\title{
Generalized Matrogenic Graphs
}

\author{
Igor E. Zverovich* \\ Rutgers Center for Operations Research, Rutgers University, Piscataway, NJ 08854-8003, USA \\ igor@ rutcor.rutgers.edu
}

Received April 29, 2002

AMS Subject Classification: 05B35, 05C62

\begin{abstract}
Using a forbidden induced subsystem characterization of matroids, we propose a method for characterizing classes of $\mathcal{P}$-matrogenic graphs for all hereditary graph properties $\mathcal{P}$.

Keywords: independence system, matroid, matrogenic graph, forbidden induced subsystem characterization
\end{abstract}

\section{References}

1. C. Benzaken and P.L. Hammer, Boolean techniques for matroidal decomposition of independence systems and applications to graphs, Discrete Math. 56 (1) (1985) 7-34.

2. G. Ding and P.L. Hammer, Matroids arisen from matrogenic graphs, Discrete Math. 165-166 (1997) 211-217.

3. S. Foldes and P.L. Hammer, On a class of matroid-producing graphs, In: Combinatorics, Vol. I, North-Holland, Amsterdam-New York, (1978) pp. 331-352.

4. S. Foldes and P.L. Hammer, Split graphs, In: Proceedings of the Eighth Southeastern Conference on Combinatorics, Graph Theory and Computing, Congr. Numer. XIX Utilitas Math., Winnipeg, Man., (1977) pp. 311-315.

5. V. Giakoumakis, On extended $P_{4}$-sparse graphs, In: 4th Twente Workshop on Graphs and Combinatorial Optimization, (1995), extended abstract.

6. P.L. Hammer and I.E. Zverovich, Splitoids, Graph Theory Notes N. Y. 46 (2004) 36-40.

7. C.T. Hoáng, Perfect graphs, Ph. D. Thesis, McGill University, 1985.

8. N.V.R. Mahadev and U.N. Peled, Threshold Graphs and Related Topics, Ann. Discrete Math. Vol 56 North-Holland, Amsterdam, 1995.

9. P. Mihók, On graphs matroidal with respect to additive hereditary properties, In: Graphs, Hypergraphs and Matroids, II, Higher College Engrg., Zielona Góra, (1987) pp. 53-64

10. U.N. Peled, Matroidal graphs, Discrete Math. 20 (3) (1977/78) 263-286.

11. R.I. Tyshkevich, O.P. Urbanovich, and I.E. Zverovich, Matroidal decomposition of a graph, In: Combinatorics and Graph Theory, Proceedings of 30th Semester 25, Banach Center Publications PWN, Warsaw, (1989) pp. 195-205.

\footnotetext{
* Partially supported by DIMACS Summer 2002 Award.
} 
12. J.L. Wu, Forest-inducing independence systems of graphs, Appl. Math. J. Chinese Univ. 6 (3) (1991) 420-426. 